\title{
Nonlocal Criteria for Brittle and Quasi-Brittle Fracture of Geomaterials and Rocks
}

\author{
Sergey Suknev ${ }^{1 *}$ \\ ${ }^{1}$ Mining Institute of the North, Siberian Branch, Russian Academy of Sciences, Yakutsk, Russia
}

\begin{abstract}
Nonlocal criteria are used for prediction materials and rock mass failure near stress concentrations (pores, faults, openings, excavations). A common property of nonlocal fracture criteria is the introduction of the intrinsic material length characterizing its microstructure, which allows one to describe the size effect in conditions of stress concentration. At the same time the scope of their application is limited to cases of brittle or quasi-brittle fracture with a small fracture process zone. To expand the scope of the criteria for cases of fracture with a developed fracture process zone, it is proposed to abandon the hypothesis of the size of this zone as a material constant, associated only with the material structure. New fracture criteria are proposed, which are the development of the average stress criterion, and point stress criterion, and which contain a complex parameter that characterizes the size of the fracture process zone and accounts not only for the material structure, but also plastic properties of the material, geometry of the sample, and its loading conditions. Expressions are obtained for the critical pressure in the problem of the formation of tensile cracks under compression in the samples of geomaterials with a circular hole. The calculation results are in good agreement with the experimental data on the fracture of drilled gypsum plates.
\end{abstract}

\section{Introduction}

Nonlocal fracture criteria are based on the concept of the formation of a fracture process zone in the material, in which there is local redistribution of stresses, whereas the main material is deformed elastically until fracture [1-8]. Typical representatives of such quasibrittle materials are geomaterials (concrete, gypsum), and rock. A general property of these criteria is the introduction of intrinsic material length that characterizes its structure, which allows describing the size effect in conditions of stress concentration, thereby expanding the scope of application in comparison with conventional criteria. Pointing to the general property of the nonlocal criteria, Taylor [9] proposes to consider them as partial expressions of some general "theory of critical distances". Recently, there have been a large number of papers that, in order to describe the fracture of materials with openings, appeal to the theory of critical distances. In 2008, a special issue of the Engineering Fracture Mechanics was devoted to this theory [10].

* Corresponding author: suknyov@igds.ysn.ru 
The scope of application of nonlocal criteria is, first of all, the brittle fracture of materials with openings. To describe ductile fracture, additional information is needed on the inelastic behavior of the material in the fracture process zone or on the material constants characterizing its plastic properties. An intermediate position between brittle and ductile fracture is occupied by quasi-brittle fracture region. Paper considers the possibility of expanding the scope of application of nonlocal criteria for cases of fracture with a developed fracture process zone.

\section{Fracture criteria}

Below, quasi-brittle fracture is understood as a sudden propagation of an unstable crack (also characteristic of brittle fracture), accompanied by the formation of a large fracture process zone. The size $d$ of the fracture process zone is not associated with the size of the crack, as is customary in fracture mechanics, but with the characteristic size $d_{0}$ of the material structure. We talk about brittle fracture if $d=d_{0}$ and ductile fracture if $d>>d_{0}$. The redistribution of stresses within the limits of $d_{0}$ is not related with the plastic (in the macroscopic sense) deformation of the material. The plastic properties of the material begin to appear when $d>d_{0}$, and the larger $d$ with respect to $d_{0}$, the more they manifest themselves. Taking this into account, we represent $d$ in the following form:

$$
d=d_{0}+\beta L_{e}
$$

Here $L_{e}$ is the size of the stress concentration zone, $\beta$ is the dimensionless parameter that characterizes the plasticity of the material. For brittle materials, $\beta=0$; for plastic materials, $\beta>>1$. In the case where $\beta \sim 1$, the material is characterized by moderate plastic properties.

For ductile fracture, the critical stress does not depend on the size of the opening, so the size of the damage zone is proportional to the size of the opening and, accordingly, to the size $L_{e}$ (under unchanged boundary conditions). In brittle fracture, on the contrary, the size of the damage zone does not depend on the size of the opening and is determined by the structure of the material.

During compression, the behavior of the failure stress characterizing the formation of tensile cracks at the opening has the form shown in Figure 1. For small values of $L_{e}$, the material does not feel the stress concentration and collapses as a smooth sample when the applied pressure reaches the compressive strength $C_{0}$. After reaching the critical size of the opening, the failure pressure $p_{c}$ decreases, asymptotically approaching the tensile strength of the material $T_{0}$ in the case of brittle failure and the stress $T_{s}\left(C_{0}>T_{s}>T_{0}\right)$ in the case of ductile fracture.

This behavior of the failure stress agrees with the current understanding of the real solid as containing primary, inherent defects. Accordingly, small artificial defects, whose sizes are comparable with the sizes of the microstructural components of the material, do not affect its strength until their sizes reach a certain (critical) value. 


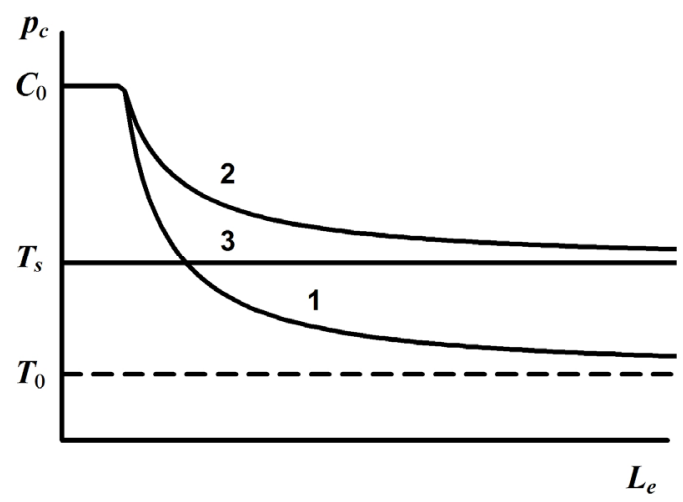

Fig. 1. Fracture diagrams: 1 - brittle fracture; 2 - quasi-brittle fracture; 3 -ductile fracture.

\subsection{Average stress criterion}

The most well-known nonlocal criterion is the average stress criterion (ASC), or integral criterion [1-3], which has the form

$$
\left\langle\sigma_{e}\right\rangle_{d}<\sigma_{0}
$$

where $\left\langle\sigma_{e}\right\rangle_{d}$ is the equivalent stress averaged at the distance $d$ along the dangerous cross section and characterizing the internal stressed state of the deformed body:

$$
\left\langle\sigma_{e}\right\rangle_{d}=\frac{1}{d} \int_{0}^{d} \sigma_{e}(x) d x .
$$

For brittle materials, the size of averaging of $d$ is assumed as a material constant, which characterizes the structure of the material: $d=d_{0}=$ const, the material strength $\sigma_{0}$ is also assumed to be a constant.

Critical pressure for the sample with a circular hole [11]:

$$
p_{c}=\chi C_{0} \frac{(1+2 d / l)^{3}}{1+d / l}, l>l_{c} .
$$

Here $\chi=T_{0} / C_{0}, l$ is the hole diameter, and $l_{c}$ is the critical diameter of the hole.

To describe the quasi-brittle fracture, we determine the size of averaging according to equation (1), in which the size of the stress concentration zone has the form

$$
L_{e}=\frac{\sigma_{e}}{\left|\operatorname{grad} \sigma_{e}\right|}
$$

The normal stress distribution $\sigma_{y}$ along the axis of loading has the form [12]:

$$
\sigma_{y}=\frac{p}{2}\left(3 \frac{a^{4}}{x^{4}}-\frac{a^{2}}{x^{2}}\right)
$$


Here $p$ is the applied compression stress (pressure), and $a$ is the hole radius. The origin is chosen at the center of the hole, and the stress $p$ is considered to be positive. The size of the stress concentration zone calculated by equation (5) with account for equation (6) is $L_{e}=l / 10$.

The critical pressure is determined by substituting equation (1) into equation (4) with account for the obtained estimate for $L_{e}$ :

$$
p_{c}=\chi C_{0} \frac{\left(1+2 d_{0} / l+0,2 \beta\right)^{3}}{1+d_{0} / l+0,1 \beta} .
$$

As $l \rightarrow \infty$, we have:

$$
\frac{T_{s}}{T_{0}}=\frac{(1+0,2 \beta)^{3}}{1+0,1 \beta}
$$

\subsection{Point stress criterion}

The application of the ASC requires the implementation of an integration procedure, which, in some cases, causes certain difficulties, especially when solving asymmetrical problems. Therefore, along with the ASC, the point stress criterion (PSC) is widely used [3]. In this criterion, the integration is replaced by calculating the equivalent stress $\sigma_{e}$ at some point that is located at the distance $d$ from the maximum point. The strength criterion takes the form

$$
\sigma_{e}(d)<\sigma_{0}
$$

The parameter $d$ is also considered to be a material constant that does not coincide with the identical parameter in the integral criterion. The critical pressure for the sample with a circular hole is determined by the equation [11]:

$$
p_{c}=\chi C_{0} \frac{2(1+2 d / l)^{4}}{3-(1+2 d / l)^{2}}, \quad l>l_{c} .
$$

Equation (1) is substituted into equation (10), and, with account for the estimate for $L_{e}$, we have:

$$
p_{c}=\chi C_{0} \frac{\left(1+2 d_{0} / l+0,2 \beta\right)^{3}}{1+d_{0} / l+0,1 \beta}
$$

As $l \rightarrow \infty$, we have:

$$
\frac{T_{s}}{T_{0}}=\frac{2(1+0,2 \beta)^{4}}{3-(1+0,2 \beta)^{2}} .
$$




\section{Experimental verification and discussion}

The applicability of the developed quasi-brittle fracture criteria can be verified by the experimental data on the tensile cracks initiation under compression in the samples of geomaterials with circular holes.

We test samples made of gypsum cement of various compositions. One series of samples is made from a gypsum cement with high (higher than $90 \%$ ) content of hemihydrate gypsum (Gypsum 1), and the second one from the gypsum cement having a low (within 60-70\%) content of hemihydrate gypsum in the initial composition (Gypsum 2 ). The peculiarities of sample preparation and the experimental procedure are given in [13, 14].

The samples from Gypsum 1 demonstrate brittle fracture. The formation of tensile cracks at the contour of the circular hole is sudden for all the investigated diameters of holes, and the crack length at the time of initiation is 5 to $6 \mathrm{~cm}$. With an increase in the hole diameter, there is a decrease in the critical pressure at the time of crack initiation, and further development of cracks leads to failure of the sample in the form of cleaving into two pieces.

The formation of tensile cracks at the contour of the circular hole in the samples of Gypsum 2 have a different character for small and large holes. Their formation on the contour of a small diameter (up to $5 \mathrm{~mm}$ inclusive) is sudden, and the samples demonstrate brittle fracture as in the samples of Gypsum 1. The formation and propagation of cracks on the contour of a hole of large diameter $(10 \mathrm{~mm}$ and larger) occurs gradually, which is typical for ductile fracture. After the formation of the remote cracks located at a distance from the hole, the opening of primary tensile cracks decreases, their growth stops, and they no longer affect the further fracture of the sample.

\subsection{Average stress criterion}

Figure 2 shows the experimental data (solid circles) on the load at the moment of tensile cracks initiation from the hole as a function of its diameter, obtained from the samples of Gypsum 1, and the results of the calculations of the critical pressure (curve 1), carried out according to equation (7) for $\beta=0$. The size $d_{0}$ is $1.1 \mathrm{~mm}$ and comparable to the size of the largest pores. The dashed line is calculated according to the conventional approach.

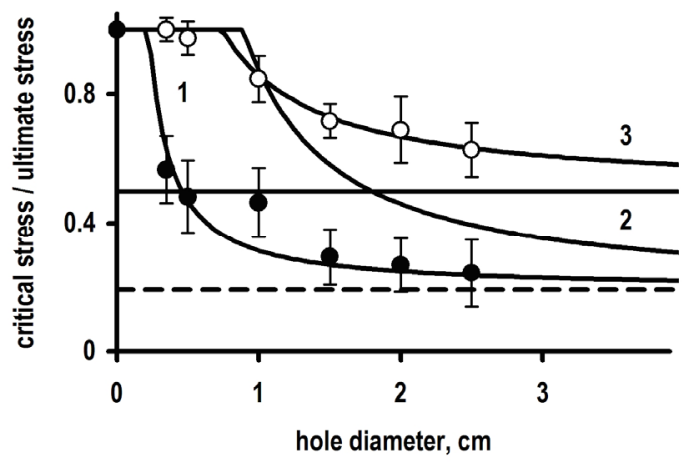

Fig. 2. Relationship of critical pressure with hole diameter. Calculated by the modified ASC.

The experimental data (open circles) and the calculation results for Gypsum 2 for $\beta=0$ (curve 2) and $\beta=2,5$ (curve 3) are also given in figure 2. The size $d_{0}$ is $4.5 \mathrm{~mm}$ in the first 
case and $2 \mathrm{~mm}$ in the second case. In accordance with equation (8), the stress $T_{S}$ is equal to $T_{0}$ in the first case and $T_{s}=2,7 T_{0}$ (solid straight line) in the second case.

Figure 2 illustrates a significant size effect, i.e. the effect of the diameter of the hole on the local strength of the material. When it decreases, the critical pressure increases, reaching the compressive strength limit. When it increases, the critical pressure asymptotically approaches the tensile strength $T_{0}$ for Gypsum 1 and the stress $T_{s}$ for Gypsum 2. This behavior is well described by the modified ASC, in which the averaging size $d$ is determined by equation (1).

\subsection{Point stress criterion}

Figure 3 illustrates the experimental data (solid circles) on the value of the load at the moment of tensile cracks initiation from the hole as a function of its diameter, obtained from the samples of Gypsum 1, and the calculation results for the critical pressure (curve 1) according to equation (11) for $\beta=0$. The size $d_{0}$ is $0.55 \mathrm{~mm}$. The dashed line is calculated according to the conventional approach.

The experimental data (open circles) and the calculation results for Gypsum 2 for $\beta=0$ (curve 2) and $\beta=1$ (curve 3) are also given in figure 3. The size $d_{0}$ is $2.25 \mathrm{~mm}$ in the first case and $0.6 \mathrm{~mm}$ in the second case. In accordance with equation (12), the stress $T_{s}$ is equal to $T_{0}$ in the first case and $T_{s}=2,7 T_{0}$ (solid straight line) in the second case.

The experimental results are well described by the modified PSC, in which the size $d$ is determined by equation (1).

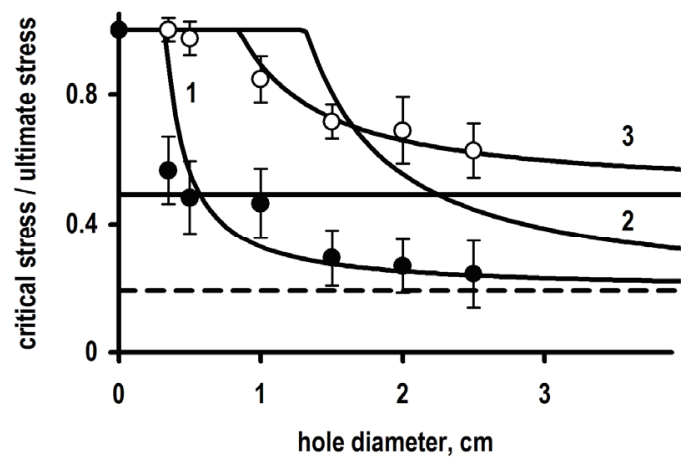

Fig. 3. Relationship of critical pressure with hole diameter. Calculated by the modified PSC.

As can be seen from figures 2 , and 3 , the experimental data obtained for brittle (Gypsum 1) and ductile (Gypsum 2) crack propagation are equally well described by the modified nonlocal criteria that take into account the change in the size of the fracture process zone in accordance with equation (1). The plasticity parameter $\beta$ is zero in the first case, and it is determined by the form of the nonlocal criterion and depends on the ratio $T_{s} / T_{0}$ in the second case. The stress $T_{s}$ is determined by the asymptotic form of the dependence $p_{c}\left(L_{e}\right)$ and equals $2,7 T_{0}$ for the criteria considered. 


\section{Conclusion}

On the basis of the analysis of existing nonlocal fracture criteria, whose distinguishing feature is the introduction of the additional material constant characterizing its structure (intrinsic material length), it is shown that the scope of their application is limited to cases of brittle or quasi-brittle fracture with a small fracture process zone. To expand the scope of application of the criteria for cases of quasi-brittle fracture with a developed fracture process zone, it is proposed to abandon the hypothesis of the size of the fracture process zone as a material constant associated only with its structure. The structural parameter underlying the nonlocal criteria combined into the "theory of critical distances" should be considered as a material constant only in one particular case, which is brittle fracture. For quasi-brittle materials, this parameter reflects not only the structural features of the material, but also plastic properties of the material, geometry of the sample, and its loading conditions.

The proposed approach is used in the development of new (modified) average stress criterion, and point stress criterion. The applicability of the developed quasi-brittle fracture criteria is verified on the problem of the tensile cracks initiation under compression in the samples of geomaterials with circular holes. It is shown that the modified criteria describe well the experimental data on the fracture of quasi-brittle materials.

The research was supported by the Russian Foundation for Basic Research under grant number 18-0500323.

\section{References}

1. H. Neuber, Kerbspannungslehre, Grundlagen für genaue Spannungsrechnung (Springer, Berlin, 1937).

2. V.V. Novozhilov, J. Appl. Math. Mech. 33, 201 (1969).

3. J.M. Whitney, R.J. Nuismer, J. Compos. Mater. 8, 253 (1974).

4. B.J. Carter, E.Z. Lajtai, Y. Yuan, Int. J. Fract. 57, 3, 221 (1992).

5. D. Radaj, S. Zhang, Eng. Fract. Mech. 50, 1, 111 (1995).

6. S.E. Mikhailov, Eng. Fract. Mech. 52, 4, 731 (1995).

7. Z. Yosibash, A. Bussiba, I. Gilad, Int. J. Fract. 125, 3-4, 307 (2004).

8. D. Taylor, P. Cornetti, N. Pugno, Eng. Fract. Mech. 72, 7, 1021 (2005).

9. D. Taylor, The theory of critical distances: a new perspective in fracture mechanics (Elsevier, Oxford, 2007).

10. Critical distance theories of fracture, Eng. Fract. Mech. 75, 1695 (2008).

11. S.V. Suknev, J. Appl. Mech. Tech. Phys. 56, 6, 1078 (2015).

12. S.P. Timoshenko, J.N. Goodier, Theory of elasticity (McGraw-Hill, New York, 1970).

13. S.V. Suknev, V.K. Elshin, M.D. Novopashin, J. Min. Sci. 39, 5, 460 (2003).

14. S.V. Suknev, J. Min. Sci. 44, 1, 43 (2008). 